\title{
PERFIL EPIDEMIOLÓGICO NA UNIDADE DE TERAPIA INTENSIVA NEONATAL E PEDIÁTRICA DE UM HOSPITAL COM ÊNFASE NA FISIOTERAPIA
}

\author{
EPIDEMIOLOGICAL PROFILE IN THE NEONATAL AND PEDIATRIC INTENSIVE CARE \\ UNIT OF A HOSPITAL WITH AN EMPHASIS ON PHYSICAL THERAPY
}

\begin{abstract}
Grazielle Corazza Sarettoํㅡ, Carla Maria Pacheco², Emerson José Corazza ${ }^{3}$, Herica Salvaro Fernandes ${ }^{4}$
\end{abstract}

\section{RESUMO}

Objetivo:Traçar o perfil epidemiológico dos pacientes internados na Unidade de Terapia Intensiva Neonatal e Pediátrica (UTI-NP) de um hospital do extremo sul catarinense, com ênfase na importância da atuação do fisioterapeuta neste ambiente. Métodos: Foram coletados os dados dos prontuários, por meio do sistema GEM-Saúde, de todos os pacientes internados na UTI-NP de um hospital do extremo sul catarinense. O período de coleta foi feito entre os meses de fevereiro e abril, referente ao período de janeiro de 2017 a janeiro de 2018. Resultados: Tendo como n 230 pacientes, o sexo prevalente foi o masculino, dentre as doenças destacou-se a prematuridade, a média dos dias de internação foi de 18,5 dias, o uso de oxigênio foi de $65,2 \%$, da ventilação mecânica não invasiva (VMNI) de $40,9 \%$ e da ventilação mecânica invasiva (VMI) de 48,7\%, 87,4\% tiveram intervenção fisioterapêutica e 9,6\% foram a óbito. Conclusão: Os resultados desse estudo podem auxiliar na elaboração de políticas de saúde.Cabe ressaltar que novas pesquisas podem ser feitas no hospital de estudo, tendo como base de dados os resultados obtidos. Sugere-se a ampliação do período de tempo de pesquisa e, aprofundando assim, os temas abordados, como por exemplo, a justificativa para o alto número de prematuros internados.

Palavras-chave:Epidemiologia; Fisioterapia; UTI neonatal e pediátrica; perfil epidemiológico.

\footnotetext{
ABSTRACT

Objective: Draw the epidemiological profile of patients hospitalized in the Neonatal and Pediatric Intensive Care Unit neonatal and pediatric (NPICU) of a hospital of the end South of

${ }^{1}$ Graduada em Fisioterapia pela Universidade do Extremo Sul Catarinense-UNESC, Criciúma/SC, Brasil. Rua Presidente Prudente de Moraes, n¹96, ed. Eagle apto 602, Santo Antônio, Joinville-SC, Brasil. CEP: 89218-000. E-mail: graziellecorazzasaretto@gmail.com, tel.: (48) 998241916.

${ }^{2}$ Graduada em Fisioterapia pela Universidade do Extremo Sul Catarinense-UNESC, Criciúma/SC, Brasil. Rua Marcelo Lodetti, nº100, ed. Imperador apto 303, Centro, Criciúma-SC, Brasil. CEP: 88801-510. E-mail: carlamp.94@gmail.com, tel.: (48) 99135-5755.

3 Professor mestre, coordenador do curso de Engenharia mecânica na Universidade da Região de JoinvilleUNIVILLE, Joinville/SC, Brasil. E-mail: ej.corazza@gmail.com, tel.: (47) 99974-0749.

${ }^{4}$ Orientadora, professora mestre do curso de Fisioterapia na Universidade do Extremo Sul Catarinense-UNESC e Fisioterapeuta do HMISC, Criciúma/SC, Brasil. E-mail: hericasalvaro@ hotmail.com, tel.: (48) 99932-7098.
} 


\section{PERFIL EPIDEMIOLÓGICO NA UNIDADE DE TERAPIA INTENSIVA NEONATAL E PEDIÁTRICA DE UM HOSPITAL COM ENFASE NA FISIOTERAPIA}

Santa Catarina, with emphasis in the importance of the physiotherapist's performance in this environments. Methods: the data of all the patients hospitalized in the NPICU of a hospital of the South of Santa Catarina were collected in the handbooks through the GEM-Saúde system. The collection period was made between the months of February and April, regarding the period of January of 2017 to January of 2018. Results: Having as n 230 patients, the sex prevalence was male. Among the diseases, the prematurity stood out, the average of the days of hospitalization was of 18,5 days, the use of oxygen was of $65,2 \%$, of the non-invasive ventilation mechanics of $40,9 \%$ and of the invasive ventilation mechanics of $48,7 \% .87,4 \%$ had physiotherapeutic intervention and 9,6\% came to death. Conclusion: The results of this study can assist in the development of public health politics. Nonetheless, it is important to emphasize that new researches can be conducted in the same hospital, having as base of data the obtained results. The period of research could be extended, going deeper in the subject studied, such as the explanation for the high number of hospitalized premature babies.

Keywords: Epidemiology; Physiotherapy; ICU neonatal and pediatric;epidemic profile.

\section{INTRODUÇÃO}

Epidemiologia é o estudo da distribuição e dos determinantes de estados ou eventos relacionados à saúde em populações específicas e suas aplicações no controle de problemas de saúde(LIMA-COSTA; BARRETO, 2003). Esta ciência analisa as características da população internada, assim como as condições clínicas e as circunstâncias em que as mortes ocorrem(LANETZKI et. al., 2012). A epidemiologia oferece medidas específicas de promoção, prevenção e recuperação da saúde individual e coletiva (BRASIL, 2005). As informações e conhecimentos reproduzidos a partir desta ciência apoia a tomada de decisão no planejamento, na administração e na avaliação de sistemas dos programas de serviços e ações de saúde (LIMA-COSTA; VERAS, 2003).

Através das informações levantadas nas unidades de terapia intensiva (UTIs) obtêmse indicadores de saúde que retratam as condições de vida da população; os níveis de mortalidade, causas e determinantes; demanda atendida pelo serviço; padrão de morbidade da população; grau de risco de um evento ou agravo em saúde; e o conhecimento do perfil sóciodemográfico da população(EINLOFT et. al.,2002).

Estudos epidemiológicos em unidade de terapia intensiva neonatal e pediátrica (UTINP) têm sido cada vez mais frequentes, tendo como objetivo auxiliar o conhecimento e compreensão do processo saúde-doença na população infantil. Além, de poder se transformar em formas de produção de indicadores necessários para o planejamento de ações em saúde (QUINTINO, 2015). A UTI-NP é a área hospitalar que reúne uma equipe qualificada com equipamentos de alta tecnologia, para que as crianças criticamente doentes ou com potencial 


\section{PERFIL EPIDEMIOLÓGICO NA UNIDADE DE TERAPIA INTENSIVA NEONATAL E PEDIÁTRICA DE UM HOSPITAL COM ENFASE NA FISIOTERAPIA}

risco de vida possam ser tratadas com eficiência (CORULLÓN, 2007). São diversas as sequelas que a UTI e a doença em si podem trazer a criança, como alterações no seu estado funcional, saúde mental, interaçãosocial, função cognitiva e grau de incapacidade(ALIEVI, 2007).

A fisioterapia nas UTIN é uma modalidade relativamente nova (MAIA, 2016), que teve inícioem pacientes pediátricos do Hospital das Clinicas da Faculdade de Medicina da Universidade de São Paulo (USP), em 1977. Surgindo após o aparecimento de novos conceitos técnicos e científicos que foram se estabelecendo na prática, diante de maiores perspectivas de sobrevida do recém-nascido de alto risco (SILVA; FORMIGA, 2010).

A presença do fisioterapeuta tem sido cada vez mais frequente nas UTIs, onde suas técnicas de atendimento são de grande importância para uma recuperação eficaz e na preservação da funcionalidade (MONDADORI et. al., 2016) sendo responsável por reabilitar, avaliar e prevenir alterações cinético funcionais (JOHNSTON et. al.,2012). No âmbito intensivistasua participação está relacionada com procedimentos complexos na UTI, tais como a ventilação artificial, o atendimento de parada cardíaca, a intubação endotraqueal, bem como a monitoração da mecânica pulmonar (COF FITO, 2014).Dentre os objetivos da assistência fisioterapêutica em recém-nascidos e em crianças, podem ser citados: adaptar o suporte ventilatório, auxiliar no desmame da ventilação mecânica (VM) e da oxigenoterapia, preservar a permeabilidade das vias aéreas, potencializar a função respiratória de modo a favorecer as trocas gasosas, adequar à relação ventilação-perfusão, prevenir e tratar as complicações pulmonares, além de atuar na parte motora (GUIMARÃES; PEREIRA, 2012; ZARA, 2016).

Sabendo da importância da determinação do perfil epidemiológico paraa melhor compreensão do processo saúde doença e para se obter indicadores de saúde, este artigo tem como objetivo traçar o perfil dos pacientes internados na UTI-NP de um hospital do extremo sul catarinense, com ênfase na importância da atuação do fisioterapeuta neste ambiente.

\section{MÉTODOS}

Trata-se de um estudo descritivo retrospectivo, com abordagem quantitativa. Esta pesquisafoi aprovada pelo Comitê de Ética em Pesquisa (CEP) da Universidade do Extremo Sul Catarinense (UNESC), Santa Catarina, Brasil com o número 2.563.870 em 26/03/2018.

Foram coletados os dados dos prontuários, por meio do sistema GEM-Saúde, de todos os pacientes internados na UTI-NP de um hospital do extremo sul catarinense. A 
unidade possui 10 vagas na UTI, sendo 7 leitos neonatais e 3 leitos pediátricos, atendendo crianças provenientes de todo o estado de Santa Catarina, de 0 a 13 anos 11 meses e 29 dias de idade.O período de coleta foi feito entre os meses de fevereiro e abril, referente ao período de janeiro de 2017a janeiro de 2018, as variáveis utilizadas foram: sexo, idade na data de internação, mês de internação, diagnóstico inicial, idade gestacional, peso ao nascimento, procedência, uso e tempo de uso deventilação mecânica invasiva (VMI) e ventilação mecânica não invasiva (VMNI), uso de Oxigênio, tratamento fisioterapêutico, permanência e óbito. Foram excluídos do estudo prontuários incompletos ou que não estiveram devidamente preenchidos

Os dados coletados foram analisados com auxílio do software IBM StatisticalPackage for the Social Sciences (SPSS) versão 23.0. As variáveis quantitativas foram expressas por meio de mediana e amplitude interquartil. As variáveis qualitativas foram expressas por meio de frequência e porcentagem. Os testes estatísticos foram realizados com um nível de significância $\alpha=0,05$ e, portanto, confiança de $95 \%$. A investigação da existência de associação entre as variáveis qualitativas foi realizada por meio da aplicação dos testes Razão de Verossimilhança.

\section{RESULTADOS}

Foram analisados 230 prontuários referentes ao período de janeiro de 2017 a janeiro de 2018, sendo todos admitidos no estudo. Destes, 119 (52\%) eram do sexo masculino e 111 (48\%) do sexo feminino. Foi verificado que não houve prevalência significativa perante o sexo.

Tabela 01 .

\begin{tabular}{|c|c|}
\hline & $\begin{array}{l}\mathrm{n}(\%) \\
\mathrm{n}=230\end{array}$ \\
\hline \multicolumn{2}{|c|}{ Intervenção Fisioterapêutica } \\
\hline Sim & $202(87,8 \%)$ \\
\hline Não & $28(12,2 \%)$ \\
\hline \multicolumn{2}{|c|}{ Diagnóstico Inicial } \\
\hline Prematuridade & $91(39,6 \%)$ \\
\hline
\end{tabular}




\begin{tabular}{lc} 
Doenças Pulmonares & $56(24,3 \%)$ \\
Sepse & $15(6,5 \%)$ \\
Doenças Cardiovasculares & $10(4,3 \%)$ \\
Síndromes & $7(3,0 \%)$ \\
Oncologia & $6(2,6 \%)$ \\
Traumas & $6(2,6 \%)$ \\
Pós-operatório & $5(2,2 \%)$ \\
Aspiração Meconial & $5(2,2 \%)$ \\
Icterícia Neonatal & $4(1,7 \%)$ \\
Outros & $25(10,9 \%)$ \\
\hline
\end{tabular}

Fonte: Dados da pesquisa 2018.

A mediana da idade foi de $1,00(0,00-2,00)$, sendo a idade mínima de 1 dia e a máxima de 13 anos. Quanto aos dias de internação, a média de duração da internação foi de 18,5 dias, variando entre 1 e 88 dias, sendo que 77,83\% das crianças permaneceram internadas de 1 a 20 dias. Dos pré-termos a média da idade gestacional foi de 32,94 semanas e a média do peso ao nascimento foi de $2.102,17$ gramas.

A distribuição das frequências por motivo de internação e o percentual de pacientes que necessitaram da intervenção fisioterapêutica, foram descritas através da Tabela 01. Evidenciou-se que 39,6\% tiveram como diagnóstico a prematuridade, seguido das doenças pulmonares $(24,3 \%)$ e sepse $(6,5 \%)$, sendo mais da metade dos pacientes. Identificamos também que 10,9\% puderam ser inscrito em outras patologias, porém, não sendo relevante ao estudo, pois se tratava de baixos percentuais quando analisados separadamente. Em relação ao tratamento fisioterapêutico realizado na UTI-NP, das 230 crianças, 202 (87,8\%) foram submetidas à fisioterapia. Destes, $147(72,7 \%)$ pacientes fizeram fisioterapia respiratória e 55 $(27,3 \%)$ fisioterapia motora, tendo crianças que fizeram tanto fisioterapia respiratória quanto motora.

Tabela 02.

\begin{tabular}{|c|c|c|c|}
\hline \multirow[t]{4}{*}{ Diagnóstico Inicial } & & & \multirow{4}{*}{ Valor-p } \\
\hline & \multicolumn{2}{|c|}{ Intervenção Fisioterapêutica } & \\
\hline & Sim & Não & \\
\hline & $n=202$ & $\mathrm{n}=28$ & \\
\hline
\end{tabular}




\begin{tabular}{|c|c|c|c|}
\hline Prematuridade & $79(86,8)$ & $12(13,2)$ & $0,809^{\dagger}$ \\
\hline Síndromes & $7(100,0)$ & $0(0,0)$ & \\
\hline Doenças Cardiovasculares & $9(90,0)$ & $1(10,0)$ & \\
\hline Doenças Pulmonares & $49(87,5)$ & $7(12,5)$ & \\
\hline Oncologia & $6(100,0)$ & $0(0,0)$ & \\
\hline Sepse & $13(86,7)$ & $2(13,3)$ & \\
\hline Pós-operatório & $4(80,0)$ & $1(20,0)$ & \\
\hline Traumas & $6(100,0)$ & $0(0,0)$ & \\
\hline Aspiração Meconial & $4(80,0)$ & $1(20,0)$ & \\
\hline Icterícia Neonatal & $3(75,0)$ & $1(25,0)$ & \\
\hline Outros & $22(88,0)$ & $3(12,0)$ & \\
\hline
\end{tabular}

†Valor obtido por meio da aplicação do teste de Razão de Verossimilhança.

Fontes: Dados da pesquisa 2018.

Na tabela 02, foi feita a relação entre a intervenção fisioterapêutica referente a cada patologia. Todas as crianças com diagnósticos de síndromes, doenças oncológicas e traumas tiveram intervenção fisioterapêutica. Das 56 crianças internadas por doenças pulmonares, apenas 7 não tiveram intervenção fisioterapêutica.

Dos meses de internação, os de maior incidência foramjaneiro de 2018 com 11,7\%, acompanhado de outubro e agosto de 2017 com 10,4\% e 8,7\%, respectivamente. Também foi analisado um acréscimo na porcentagem de janeiro de 2018 em relação a janeiro de 2017, sendo $11,7 \%$ e $7,4 \%$, respectivamente.

O hospital onde foi realizado o estudo recebe pacientes de todas as regiões do estado catarinense. A procedência das crianças foi dividida por seis regiões, sendo a grande maioria do sul catarinense $(95,2 \%)$, seguido por norte catarinense $(1,7 \%)$, oeste catarinense $(1,3 \%)$, grande Florianópolis $(0,9 \%)$, região serrana $(0,4 \%)$ e vale do Itajaí $(0,4 \%)$. 
Figura 01: Tipos de equipamentos utilizados.

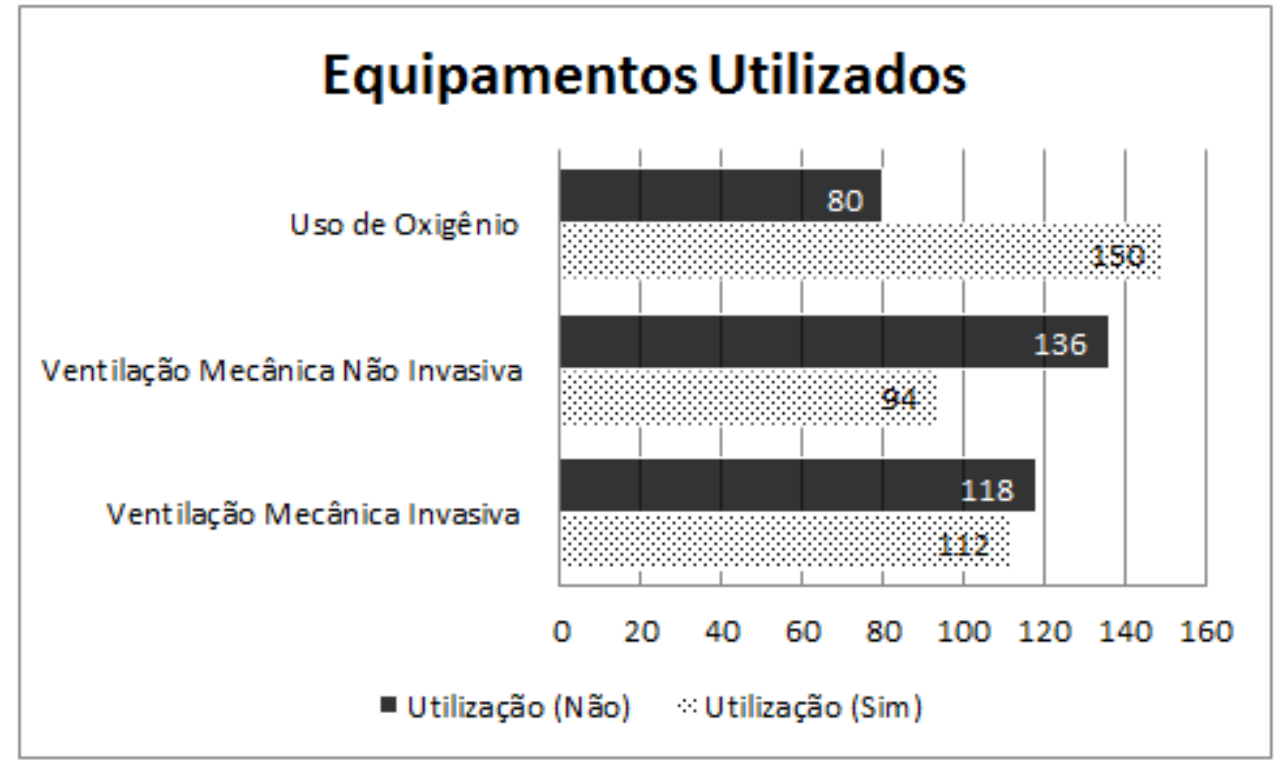

Fontes: Dados da pesquisa 2018.

Tabela 03

$$
\mathrm{n}(\%)
$$

Diagnóstico Inicial

Ventilação Mecânica Invasiva

$\begin{array}{ccc}\text { Sim } & \text { Não } & \text { Valor-p } \\ \mathrm{n}=112 & \mathrm{n}=118\end{array}$

\begin{tabular}{lccc}
\hline Prematuridade & $45(49,5)$ & $46(50,5)$ & $0,435^{\dagger}$ \\
Doenças Pulmonares & $26(46,4)$ & $30(53,6)$ & \\
Sepse & $11(73,3)$ & $4(26,7)$ & \\
Doenças Cardiovasculares & $4(40,0)$ & $6(60,0)$ & \\
Síndromes & $5(71,4)$ & $2(28,6)$ & \\
Oncologia & $2(33,3)$ & $4(66,7)$ \\
Traumas & $4(66,7)$ & $2(33,3)$ \\
Pós-operatório & $3(60,0)$ & $2(40,0)$ \\
Aspiração Meconial & $2(40)$, & $3(60,0)$ \\
Icterícia Neonatal & $1(25,0)$ & $3(75,0)$ & \\
Outros & $9(36,0)$ & $16(64,0)$
\end{tabular}

†Valor obtido por meio da aplicação do teste de Razão de Verossimilhança.

Fontes: Dados da pesquisa 2018. 
O percentual do uso de recursos para promoção da melhor ventilação dos pacientes internados foi descrito na Figura 01. Dos equipamentos utilizados para melhora da oxigenação das crianças em $65,2 \%$ dos casos foram utilizados o oxigênio, em 40,9\% a VMNI e em $48,7 \%$, a VMI. O n referente ao uso de oxigênio foi de 150, do uso da VMI foi de 112 e o da VMNI 94 crianças. A mediana do tempo de ventilação em dias foi de 5,00 (3,00-8,00) para VMI e de 2,00 (1,00-3,00) para VMNI.

Na tabela 03 e 04, realizou-se o cruzamento entre a VMI e VMNI em relação ao diagnóstico inicial. Verificou-seque a mesma quantidade de crianças com doenças pulmonares que precisaram da VMI, também utilizaram a VMNI. Os valores obtidos na relação do diagnóstico com o tipo de VM utilizado na criança, não foram relevantes para o estudo.

Tabela 04

$$
\text { n }(\%)
$$

\begin{tabular}{lccc} 
& \multicolumn{2}{c}{ Ventilação Mecânica Não Invasiva } & \multirow{2}{*}{ Valor-p } \\
\cline { 2 - 3 } & Sim & Não & \\
& $\mathrm{n}=94$ & $57(62,6)$ & \\
Prematuridade & $34(37,4)$ & $30(53,6)$ & \\
Doenças Pulmonares & $26(46,4)$ & $12(80,0)$ \\
Sepse & $3(20,0)$ & $7(70,0)$ \\
Doenças Cardiovasculares & $3(30,0)$ & $1(14,3)$ \\
Síndromes & $6(85,7)$ & $4(66,7)$ \\
Oncologia & $2(33,3)$ & $1(16,7)$ \\
Traumas & $5(83,3)$ & $2(40,0)$ \\
Pós-operatório & $3(60,0)$ & $2(40,0)$ \\
Aspiração Meconial & $3(60,0)$ & $3(75,0)$ \\
Icterícia Neonatal & $1(25,0)$ & $17(68,0)$ \\
Outros & $8(32,0)$ &
\end{tabular}

†Valor obtido por meio da aplicação do teste de Razão de Verossimilhança.

Fontes: Dados da pesquisa 2018.

Em relação aos óbitos, no presente estudo, 9,6\% (22) das crianças foram a óbito, $59,1 \%$ do sexo feminino e $40,9 \%$ do sexo masculino, sendo as principais causas 
prematuridade, síndrome de Down e sepse. A prevalência da prematuridade foi no sexo masculino e a da síndrome de Down e sepse no sexo feminino.

\section{DISCUSSÃO}

A pesquisa foi realizada com o intuito de traçar o perfil epidemiológico dos pacientes internados na UTI-NP de um hospital do extremo sul catarinense referente ao período de janeiro de 2017 a janeiro de 2018.

A prevalência do sexo masculino em nosso estudo pode estar relacionada com a taxa de natalidade, que segundo o levantamento do ano de 2010do Instituto Brasileiro de Geografia e Estatísticafoi de 6.117 para o sexo masculino e de 6.002 para o sexo feminino. Em estudos comparativos foi verificado que a maioria teve como prevalência o sexo masculino (LANETZKI et. al., 2011; QUINTINO, 2015; CORULLÓN, 2007; SELESTRIN et. al., 2007; PICCOLI et. al., 2012).

A média elevada de dias de internação em relação aos outros estudos explica-se pelo fato da prevalência de prematuros, onde o baixo peso e o desenvolvimento corporal necessitam de maior tempo de internação e, pela falta de uma unidade semi-intensivano hospital onde foi realizada a pesquisa, o que incide em maior tempo de permanência de crianças com cuidados intermediários na UTI-NP(LANETZKI et. al., 2011; QUINTINO, 2015; CORULLÓN, 2007; MOLINA et. al., 2007).

Dos resultados encontrados, os principais diagnósticos foram prematuridade $(39,6 \%)$, doenças pulmonares $(24,3 \%)$ e sepse $(6,5 \%)$, discernindo do motivo de internação de outros estudos, onde os principais diagnósticos foram doenças respiratórias, acompanhado das anomalias congênitas, lesões e envenenamento (LANETZKI et. al., 2011; MOLINA et. al., 2007;VASCONCELOS, ALMEIDA, BEZERRA, 2011).Das patologias respiratórias, o desconforto respiratório e a pneumonia foram os que mais prevaleceram(LANETZKI et. al., 2011). O fato de as doenças respiratórias junto com a prematuridade serem a maioria do $\mathrm{n}$ utilizado na pesquisa dá-se pela localização do hospital, onde o frio é mais intenso, e pelo fato dogrande número de nascidos pré-termo (OLIVEIRA et. al., 2015; PICCOLI et. al., 2012; GRANZOTTO et. al., 2012). É importante ressaltar que o hospital onde foi realizado o estudo é $100 \%$ SUS, porém de acordo com as adequações viabilizadas pelo Ministério da Saúde, não há um acompanhamento adequado das gestantes durante a gravidez, faltando orientações e auxílios no período gestacional levando ao alto índice de prematuridade (OLIVEIRA et. al., 2015; BRASIL, 2006; NUNES et. al., 2016).Dentre todos os problemas respiratórios do recém-nascido pré-termo, a síndrome do desconforto respiratório é considerada uma das mais 


\section{PERFIL EPIDEMIOLÓGICO NA UNIDADE DE TERAPIA INTENSIVA NEONATAL E PEDIÁTRICA DE UM HOSPITAL COM ENFASE NA FISIOTERAPIA}

frequentes, além disso, pode-se citar a displasia broncopulmonar uma das complicações em longo prazo do sistema respiratório, devido à prematuridade (EBSERH,2016).

Nos números de óbitos do presente estudo, o sexo feminino foi o que prevaleceu. Nos demais estudos comparados, o sexo masculino foi o prevalente (LANETZKI et. al., 2011; QUINTINO, 2015; MOLINA et. al., 2007, ALMEIDA, RODRIGUES, SALGADO, 2010). Quanto às patologias, os óbitos por prematuridade destacaram-se no sexo masculino. Almeida, Rodrigues e Salgado(2010)relatam que os meninos são mais vulneráveis a alterações pré e perinatais, são abortados espontaneamente com maior frequência e mais suscetíveis a malformações congênitas. No estudo realizado por Quintino (2015), os óbitos foram de $100 \%$ na UTI neonatal e 76,78\% na UTI pediátrica, onde a mortalidade por doenças respiratórias foi mais frequente em crianças menores de cinco anos e do sexo masculino. Em outro estudo foi verificado que as maiores causas de óbito foram as afecções do aparelho respiratório $(31,03 \%)$ e que a mortalidade foi mais frequente entre os lactentes $(55,17 \%)$ (MOLINA et. al., 2007).Apesar do n de óbitos na pesquisa não ser relevante, segundo o IBGE (2011), na região sul de Santa Catarina, a taxa de mortalidade é de10,46 óbitos/1.000 nascidos vivos, sendo que a cidade onde se localiza o hospital do estudo, é a $5^{a}$ cidade com maior número de mortalidade infantil da microrregião e a $128^{\mathrm{a}}$ cidade do estado.

No estudo de Vasconcelos, Almeida e Bezerra (2011), o aumento dos atendimentos fisioterapêuticos foi significativo entre o ano de 2005 e de 2007, sendo realizado em $61,8 \%$ e em 94,1\% dos casos nos respectivos anos. Guimarães e Pereira (2012) realizaram um estudo em uma UTI neonatal em cinco hospitais do Distrito Federal com o intuito de saber quais as técnicas mais utilizadas pelos fisioterapeutas, onde se descobriu que a aspiração traqueal e de vias aéreas foi unanimidade, outras técnicas também utilizadas são vibração, bag squeezing e administração de surfactante. As indicações de intervenção fisioterapêutica e os tipos de condutas utilizadas variam de acordo com o local e o preparo técnico do profissional (VASCONCELOS, ALMEIDA, BEZERRA, 2011). No estudo de Silva e Formiga (2010), realizado na UTIN dos hospitais da cidade de Goiânia, todos os fisioterapeutas utilizaram a fisioterapia respiratória associada com a fisioterapia motora, sendo as técnicas de vibrocompressão e aspiração para higiene brônquica e, compressão-descompressão como técnica de reexpansão pulmonar. No que se refere à ventilação mecânica, todos realizaram a regulação e mudança dos parâmetros do ventilador, extubação, CPAP, BIPAP e outros. Em relação à fisioterapia motora, a mobilização passiva foi o procedimento mais usado pelos profissionais (SILVA, FORMIGA, 2010). 
Dos equipamentos utilizados para melhora da oxigenação das crianças, o oxigênio, a VMNI e a VMI são utilizados no hospital do estudo, ressaltando a importância da recomendação da utilização de desmame ventilatórionas patologias em geral (MEDEIROS, 2011). Em um estudo comparativo, onde foi correlacionadaà utilização da ventilação mecânica no ano de 2007 em relação ao ano de 2005, foi verificado que a média do tempo de ventilação não invasiva em 2005 foi de 32,8 horas e em 2007 de 34,5 horas, evidenciando que no ano de 2007 houve maior utilização de suporte ventilatório devido ao aumento de nascidos prematuros e de extremo baixo peso, e pelo fato destes pacientes não possuírem estrutura e função pulmonar adequada (VASCONCELOS, ALMEIDA, BEZERRA, 2011).Em outra pesquisa, destacou-se que a síndrome do desconforto respiratório, taquipneia transitória do $\mathrm{RN}$, displasia broncopulmonar e apneia foram aspatologias respiratórios mais frequentes citados pelos fisioterapeutas como indicações ao uso da VMNI na UTI neonatal. Sendo a média da utilização dessa ventilação o período de 24 a 72 horas (LEÃO, VIEIRA, PEREIRA, 2013).

\section{CONCLUSÃO}

O perfil traçado na pesquisa foi semelhante aos demais estudos comparativos, discernindo em algumas diretrizes como tempo de internação, diagnóstico inicial e motivo do óbito. Os resultados desse estudo podem auxiliar na elaboração de políticas de saúde. Cabe ressaltar que novas pesquisas podem ser feitas no hospital de estudo, tendo como base de dados os resultados obtidos. Sugere-se a ampliação do período de tempo de pesquisa e, aprofundando assim, os temas abordados, como por exemplo, a justificativa para o alto número de prematuros internados.

\section{REFERÊNCIAS}

ALIEVI, Patrícia Tollens. Estudo sobre o impacto da internação em crianças admitidas em Unidade de Terapia Intensiva Pediátrica. 2007. 130 f. Dissertação (Mestrado) - Curso de Medicina, Programa de Pós-Graduação em Ciências Médicas: Pediatria, Universidade Federal do Rio Grande do Sul, Porto Alegre, 2007.

ALMEIDA, Chistiana Gonçalves Meira de; RODRIGUES, Olga Maria Piazentin Rolim; SALGADO, Manoel Henrique. Diferenças no desenvolvimento de meninos e meninas em condições de risco.Boletim de Psicologia, [S. L.], v. 62, n. 136, p. 1-14, jun. 2012. 
BRASIL. Ministério da Saúde Brasil. Secretaria de Atenção À Saúde. Departamento de Ações Programáticas Estratégicas. Área Técnica de Saúde da Mulher. (comp.). Pré-natal e puerpério: atenção qualificada e humanizada - manual técnico. 3. ed. Brasília: Ministério da Saúde, 2006. 163 p. (Série A. Normas e Manuais Técnicos) - (Série Direitos Sexuais e Direitos Reprodutivos - Caderno $n^{\circ} 5$ ).

BRASIL. Ministério da Saúde, Secretaria de Vigilância em Saúde. Curso básico de vigilância epidemiológica. Brasília: Ministério da Saúde, 2005. 210 p.

CONSELHO FEDERAL DE FISIOTERAPIA E TERAPIA OCUPACIONAL (BR). Decreto Lei $\mathrm{n}^{\circ}$ 938, de 13 de novembro de 1969. Provê sobre as profissões de fisioterapeuta e terapeuta ocupacional, e dá outras providências. Os Ministros da Marinha de Guerra, do Exército e da Aeronáutica Militar, usando das atribuições que lhes confere o artigo $1^{\circ}$ do Ato Institucional $\mathrm{n}^{\mathrm{o}} 12$, de 31 de agosto de 1969 , combinado com o $\S 1^{\circ}$ do artigo $2^{\circ}$ do Ato Institucional $n^{\circ}$ 5, de 13 de dezembro de 1968, decretam. Brasília, DF, 10 jul. 2014.

CORULLÓN, Juliana Lebsa.Perfil epidemiológico de uma UTI pediátrica no sul do Brasil. 2007. 84 f. Dissertação (Mestrado) - Curso de Medicina, Programa de Pós-Graduação em Medicina/pediatria e Saúde da Criança. Mestrado em Saúde da Criança, Pontifícia Universidade Católica do Rio Grande do Sul, Porto Alegre, 2007.

EINLOFT, Paulo Roberto; GARCIA, Pedro Celiny; PIVA, Jefferson Pedro; BRUNO, Francisco; KIPPER, Délio José; FIORI, Renato M. Perfil epidemiológico de dezesseis anos de uma unidade de terapia intensiva pediátrica. Revista de Saúde Pública, [S.L.], v. 36, n. 6, p. 728-733, dez. 2002. FapUNIFESP (SciELO).http://dx.doi.org/10.1590/s003489102002000700011.

EBSERHEmpresa Brasileira de Serviços Hospitalares - Ministério da Educação Brasil. Unidade de Reabilitação do Hospital de Clínicas da Universidade Federal do Triângulo Mineiro. Atuação da Fisioterapia no Recém Nascido Prematuro - Unidade de Reabilitação do Hospital de Clínicas da Universidade Federal do Triângulo Mineiro Uberaba Minas Gerais: Ebserh, 2016. 17p.

FREIRE, Izaura Luzia Silvério; MENEZES, Luzia Clara Cunha de; SOUSA, Núbia Maria Lima de; ARAÚJO, Rhayssa de Oliveira e; VASCONCELOS, Quinidia Lúcia Duarte de Almeida Quithé; TORRES, Gilson de Vasconcelos. Epidemiologia das Infecções Relacionadas à Assistência à Saúde em Unidade de Terapia Intensiva Pediátrica.Revista Brasileira de Ciências da Saúde - Uscs, [S.L.], v. 11, n. 35, p. 9-15, jan./mar. 2013. USCS Universidade Municipal de São Caetano do Sul.http://dx.doi.org/10.13037/rbcs.vol11n35.1675.

GRANZOTTO, José A.; MOTA, Denise M.; REAL, Raul F.; DIAS, Caroline M.; TEIXEIRA, Renato F.; MENTA FILHO, João C.; TIECHER, Gabriele B.; PILECCO, Arnaldo Junior de Lima; GONÇALVES, Eduardo Rodrigues. Análise do perfil epidemiológico das internações em uma unidade de terapia intensiva neonatal. Revista da Amrigs, [Porto Alegre], v. 56, n. 4, p. 304-307, 1 out./dez. 2012. 
GUIMARÃES, Isabelle Salgado Silva; PEREIRA, Silvana Alves. A atuação do fisioterapeuta em unidade de terapia intensiva neonatal nos hospitais da rede pública do Distrito Federal.Revista Eletrônica Saúde e Ciência, [S. L.], v. 2, n. 2, p. 9-18, jul./dez. 2012.

IBGEInstituto Brasileiro de Geografia e Estatística. Ministério do Planejamento, Orçamento e Gestão.Censo Demográfico 2010: características da população e dos domicílios. Área territorial brasileira.Rio de Janeiro: IBGE, 2011. 270 p.

JOHNSTON, Cíntia; ZANETTI, Nathalia Mendonça; COMARU, Talitha; RIBEIRO, Simone Nascimento dos Santos; ANDRADE, Lívia Barboza de; SANTOS, Suzi Laine Longo dos. I Recomendação brasileira de fisioterapia respiratória em unidade de terapia intensiva pediátrica e neonatal.Revista Brasileira de Terapia Intensiva, [S.L.], v. 24, n. 2, p. 119-129, jun. 2012. GN1 Genesis Network.http://dx.doi.org/10.1590/s0103-507x2012000200005.

LANETZKI, Camila Sanches; OLIVEIRA, Carlos Augusto Cardim de; BASS, Lital Moro; ABRAMOVICI, Sulim; TROSTER, Eduardo Juan. The epidemiological profile of Pediatric IntensiveCare Center at Hospital Israelita Albert Einstein.Einstein (São Paulo), [S.L.], v. 10, n. 1, p. 16-21, mar. 2012. FapUNIFESP (SciELO).http://dx.doi.org/10.1590/s167945082012000100005 .

LEÃO, Eryka Virginia Vasconcelos; VIEIRA, Martina Estevam Brom; PEREIRA, Silvana Alves. Perfil da utilização do CPAP na UTI neonatal e o protagonismo do fisioterapeuta.Revista Movimenta, [S. L.], v. 6, n. 1, p. 386-397, jan. 2013.

LIMA-COSTA, Maria Fernanda; BARRETO, Sandhi Maria. Tipos de estudos epidemiológicos: conceitos básicos e aplicações na área do envelhecimento.Epidemiologia e Serviços de Saúde, [S.L.], v. 12, n. 4, p. 189-201, dez. 2003. Instituto Evandro Chagas.http://dx.doi.org/10.5123/s1679-49742003000400003.

LIMA-COSTA, Maria Fernanda; VERAS, Renato. Saúde pública e envelhecimento.Cadernos de Saúde Pública, [S.L.], v. 19, n. 3, p. 700-701, jun. 2003. FapUNIFESP (SciELO).http://dx.doi.org/10.1590/s0102-311x2003000300001.

MAIA, Francisco Eudison da Silva. A fisioterapia nas unidades de terapia intensiva neonatal.Revista da Faculdade de Ciências Médicas de Sorocaba, [S.L.], v. 18, n. 1, p. 6465, 1 mar. 2016. Pontifical CatholicUniversityofSão Paulo (PUC-

SP).http://dx.doi.org/10.5327/z1984-4840201622134.

MEDEIROS, Juliana Karina Brugnolli. Desmame da ventilação mecânica em pediatria.Assobrafir Ciência, [S. L.], v. 2, n. 1, p. 57-64, jun. 2011.

MEDEIROS, Laysa Gabrielle Silva; OLIVEIRA, Francimery Costa Santos de; GUIMARÃES, Jussara Pereira; NASCIMENTO, Indianara Maria Araújo do. Fisioterapia respiratória em terapia intensiva neonatal.Revista Brasileira de Educação e Saúde, Pombal, PB, v. 3, n. 3, p. 14-19, jul./set. 2013.

MOLINA, Rosemeire Cristina Moretto; MARCON, Sonia Silva; UCHIMURA, Taqueco Teruya; LOPES, Evaldo Pereira. Caracterização das internações em uma unidade de terapia intensiva pediátrica, de um hospital-escola da região sul do Brasil.Ciência, Cuidado e Saúde, 
[S.L.], v. 7, n. 1, p. 112-120, 31 dez. 2008. Universidade Estadual de Maringá.http://dx.doi.org/10.4025/cienccuidsaude.v7i0.6581.

MONDADORI, Aléxia Gabrielly; ZENI, Emanuelly de Moraes; OLIVEIRA, Alani de; SILVA, Cristiane Cosmo da; WOLF, Vaneza Lira Waldow; TAGLIETTI, Marcelo. Humanização da fisioterapia em Unidade de Terapia Intensiva Adulto: estudo transversal.Fisioterapia e Pesquisa, [S.L.], v. 23, n. 3, p. 294-300, set. 2016. FapUNIFESP (SciELO).http://dx.doi.org/10.1590/1809-2950/16003123032016.

NUNES, Juliana Teixeira; GOMES, Keila Rejane Oliveira; RODRIGUES, Malvina Thaís Pacheco; MASCARENHAS, Márcio Denis Medeiros. Qualidade da assistência pré-natal no Brasil: revisão de artigos publicados de 2005 a 2015.Cadernos Saúde Coletiva, [S.L.], v. 24, n. 2, p. 252-261, jun. 2016. FapUNIFESP (SciELO).http://dx.doi.org/10.1590/1414$462 \times 201600020171$.

OLIVEIRA, Caroline de Sousa; CASAGRANDE, Gabriela Ay; GRECCO, Luanda Collange; GOLIN, Marina Ortega. Perfil de recém-nascidos pré-termo internados na unidade de terapia intensiva de hospital de alta complexidade.Abcs Health Sciences, [S.L.], v. 40, n. 1, p. 28-32, 3 maio 2015. NEPAS.http://dx.doi.org/10.7322/abcshs.v40i1.700.

PICCOLI, Alana; SOARES, Cátia Rejane Soares; COSTA, Gabriela; SILVEIRA, Josiele Larger; FIATT, Marciane Pesamosca; CUNHA, Rosângela Symanski da. Perfil clínico de neonatos de muito baixo peso internados em uma Unidade de Tratamento Intensivo Neonatal.Revista Hcpa, [S. L.], v. 32, n. 4, p. 412-419, Não é um mês valido! 2012.

QUINTINO, Jéssica Chagas.Perfil epidemiológico de crianças internadas em UTI neonatal e pediátrica do Hospital Infantil Joana de Gusmão (SC). 2015. 53 f. TCC (Graduação) - Curso de Fonoaudiologia, Centro de Ciências da Saúde, Universidade Federal de Santa Catarina, Florianópolis, 2015.

SELESTRIN, Cláudia de Castro; OLIVEIRA, Adriana Gonçalves de; FERREIRA, Celso; SIQUEIRA, Arnaldo Augusto Franco de; ABREU, Luiz Carlos de; MURAD, Neif. Avaliação dos parâmetros fisiológicos em recém-nascidos pré-termo em ventilação mecânica após procedimentos de fisioterapia neonatal.JournalOfHumanGrowthAndDevelopment, [S.L.], v. 17, n. 1, p. 146-155, 1 abr. 2007. NEPAS.http://dx.doi.org/10.7322/jhgd.19823.

SILVA, Ana Paula Pereira; FORMIGA, Cibelle Kayenne Martins Roberto. Perfil e características do trabalho dos fisioterapeutas atuantes em Unidade de Terapia Intensiva neonatal na cidade de Goiânia - GO.Revista Movimenta, [S. L.], v. 3, n. 2, p. 62-68, mar. 2010 .

VASCONCELOS, Gabriela Arruda Reinaux de; ALMEIDA, Rita de Cássia Albuquerque; BEZERRA, Andrezza de Lemos. Repercussões da fisioterapia na unidade de terapia intensiva neonatal.Fisioterapia em Movimento, [S.L.], v. 24, n. 1, p. 65-73, mar. 2011. FapUNIFESP (SciELO).http://dx.doi.org/10.1590/s0103-51502011000100008.

ZARA, Mariana Guimarães.A atuação do fisioterapeuta em Unidades de Terapia Intensiva neonatais. 2016. 15 f. Monografia (Especialização) - Curso de Fisioterapia 
Pediátrica e Neonatal da UTI e Reabilitação Neurológica, Centro de Estudos Avançados e Formação Integrada - Ceafi, Cuiabá, 2016. 